\title{
Real Time Identification of Railway Track Surface Faults using Canny Edge Detector and 2D Discrete Wavelet Transform
}

\author{
Ali Akbar Shah", Bhawani S. Chowdhry', Tayab D. Memon ${ }^{1}$, Imtiaz H. Kalwar ${ }^{2}$ and J. \\ Andrew Ware ${ }^{3, *}$ \\ ${ }^{1}$ Mehran University of Engineering \& Technology, Pakistan \\ shah.aliakber32@gmail.com; bsc itman@yahoo.com; tayabdin82@gmail.com \\ 2DHA Suffa University, Pakistan \\ imtiaz.hussain@dsu.edu.pk \\ ${ }^{3}$ University of South Wales, UK \\ andrew.ware@southwales.ac.uk \\ *Correspondence: andrew.ware@southwales.ac.uk
}

Received: 25 ${ }^{\text {th }}$ February 2020; Accepted: 20 $0^{\text {th }}$ March 2020; Published: $1^{\text {st }}$ April 2020

Abstract: Usually, railway accidents are caused by train derailment, the mechanical failure of tracks, such as broken rails often caused by lack of railway condition monitoring. Such monitoring could identify track surface faults, such as squats, that act as a catalyst for the track to crack and ultimately break. The research presented in this paper enables real-time identification of railway track faults using image processing techniques such as Canny edge detection and 2D discrete wavelet transformation. The Canny edge detection outperforms traditional track damage detection techniques including Axle Based Acceleration using Inertial Measurement Units and is as reliable as Fiber Bragg Grating. The Canny edge detection employed can identify squats in real-time owing to its specific threshold amplitude using a camera module mounted on a specially designed handheld Track Recording Vehicle (TRV). The 2D discrete wavelet transformation validates the insinuation of the Canny edge detector regarding track damage and furthermore determines damage severity, by applying high sub band frequency filter. The entire algorithm works on a Raspberry Pi $3 \mathrm{~B}+$ utilizing an OpenCV API. When tested using an actual rail track, the algorithm proved reliable at determining track surface damage in real-time. Although wavelet transformation performs better than Canny edge detection in terms of determining the severity of track surface damage, it has processing overheads that become a bottleneck in real-time. To overcome this deficiency a very effective two-stage process has been developed.

Keywords: Railway condition monitoring; real-time; Canny edge detection; Wavelet transformation; squats 


\section{Introduction}

Most train accidents are caused by derailments [1][2][3]. In 2019, Pakistan alone recorded over a hundred train accidents the majority of which were caused by derailment [4]. While there are many causes of these derailments, most are due to negligence in track condition monitoring [5][6]. There is, therefore, a need for techniques that can effectively facilitate condition monitoring and hence enable the mitigation of the causes of derailment [6][7][8]. One common technique involves determining track damage using Inertial Measurement Units (IMUs) [2][9], mounted on the surface of Track Recording Vehicles [10][11]. However, the problem with these types of sensors is their unpredictable nature in sensing the noise component as the track gets damaged.

While devices such as accelerometers, ultrasonic, and temperature sensors, can be used for determining track damage they are often slow in processing and can provide inaccurate data [9][12]. Because of this, several other techniques are being developed such as Fiber Bragg Grating (FBG) and different types of image processing algorithms [13]. As the FBG technique are expensive and require the entire railway system to be renovated [14], its implementation is often not practical, particularly in developing countries, due to budget limitations. Therefore, in this research cost effective image processing algorithms are implemented (mainly for use in developing countries) using the Python programming language.

Various image processing techniques can be implemented for the diagnosis of rail damage, including wavelet transformation. Although such techniques allow for the severity of damage to be determined, they often cannot do so in real-time [15][16]. Thus, a faster image processing algorithm is required. Various image processing algorithms were tested and it was noted that the most reliable amongst them is the Canny edge detector [17][18], as it enables the edges of the railway track to be analyzed with almost zero delays [19]. Therefore, in this research a very effective two-stage process has been developed for overcoming this deficiency. Firstly, Canny edge detection was used for identifying track damage. Secondly, images of track where damage was identified were subsequently processed using 2D wavelet transformation. Implementation of this cost effective technique enabled the damage severity of the track to be determined in an accurate and timely fashion. Almost 10,000 different track images were tested, and the effectiveness of the two-stage process was demonstrated. Images were collected in real-time using a motorized track recording vehicle over a one week time period.

\section{Recent Developments in Railway Condition Monitoring}

Railway track condition monitoring using track recording vehicles (TRV) is an important technical challenge. Worldwide, various track recording vehicles are still in development using IMUs and image processing [11]. The information gathered is recorded and the necessary steps are taken as per maintenance precautions [10]. Several parameters are detected and reported in the literature using the acquired acceleration data. Weston et al. used bogie mounted sensors for elaborating the track irregularity [20]. Alfi et al. similarly used onboard measurements for the estimation of the wavelength track irregularities [21]. Track faults are mostly analyzed using car body mounted sensors but most of those track faults are introduced due to track surface flaws such as squats and turn out frogs [22]. These track surface faults such as squat and turn out frog occur mostly due to the sudden brakes and lack of track ballast (the ballast filling under the track) that leads to a dip angle. The dip angle is a major reason for train derailments. 
One effective methodology for obtaining optimal results when analyzing dip angle caused by track surface faults is Fiber Bragg Grating (FBG) [14]. The problem with FBG is its cost and fact that it is very hard to install because optical fiber needs to be passed underneath the track [13]. For a developing country, the cost can be unaffordable and hence other techniques need to be explored. One such technique, involves recent advancements in image processing enabled by APIs such as TensorFlow and OpenCV.

\section{Implementation of Image Processing For Track Fault Detection}

\subsection{Available Technologies}

As most of the information in an image is retained in its grayscale format, which is also quicker to process, the RGB (Red, Green, Blue) image is first converted to grayscale, using Equation 1 [17]:

$$
\text { Gray }=0.299(R)+0.587(G)+0.114(B) \quad 1
$$

Images, particularly if captured using a webcam, can be affected by various noises that might cause error propagation in the color space conversion. Thus, to reduce this noise the image edge is distributed using a two-dimensional Gaussian filter that calculates the pixel transformation using the normal distribution. The Gaussian function filter equation is used for the pixel transformation of the image into the normal distribution $\mathrm{h}(\mathrm{x}, \mathrm{y})$ :

$$
h(x, y)=\frac{1}{2 \pi \sigma^{2}} e^{-\frac{x^{2}+y^{2}}{2 \sigma^{2}}}
$$

The standard deviation that is used in the normal distribution is represented using rho. The Sobel filter mask is implemented after the completion of the image smoothing for determining the gradient value of each pixel in the captured image using a 3D horizontal mask hx and vertical mask hy as shown in equations 3 and 4, respectively:

$$
\begin{aligned}
& h_{x}=\begin{array}{ccc}
-1 & -2 & -1 \\
0 & 0 & 0 \\
1 & 2 & 1
\end{array} \\
& h_{y}=\begin{array}{lll}
-1 & 0 & 1 \\
-2 & 0 & 0 \\
-1 & 0 & 1
\end{array}
\end{aligned}
$$

The gradient value and angle are computed by obtaining the gradient values of horizontal and vertical directions, respectively:

$$
\begin{aligned}
& \operatorname{grad} f=\operatorname{mag}(\nabla G)=\left[G_{x}^{2}+G_{y}^{2}\right]^{\frac{1}{2}} \\
& \quad \theta=\tan ^{-1} \frac{G_{y}}{G_{x}}
\end{aligned}
$$

The alteration between the pixels can be observed when using the gradient amplitude obtained using equation 5. Nonzero gradient amplitude is considered as an effective block texture feature. Therefore, with the variation of gradient amplitude, the noise can be eradicated considering the smaller gradient amplitude as the noise by adjusting the Canny edge detector with a fixed threshold value. As per the research requirement, the threshold value of the gradient amplitude is kept between 0 - and 200-pixel values so that valid texture features of the railway track can be retained. 


\subsection{Discrete 2D Wavelet Transformation of an Image}

To analyze the severity of the surface damage of the railway track, the image of damage is processed with multilevel two-dimensional discrete wavelet transformation which decomposes the image into two sub bands namely: low frequency and high frequency. Almost all of the edge background details are classified into the high frequency sub band by the wavelet transformation whereas noises and approximated signal are classified into the low frequency sub band. The wavelet expansion [23] is denoted in equation 7.

$$
f(t)=\sum_{k} c_{j, k} \emptyset_{j, k}(t)+\sum_{j} \sum_{k} d_{j, k} \varphi_{j, k}(t)
$$

Projection of the $\mathrm{f}(\mathrm{t})$ on the scale space is achieved using equation 7 , whereas the decomposition form of the $f(t)$ is achieved using equations 8 and 9 .

$$
\begin{aligned}
& c_{j, k}==\left(f(x), \emptyset_{j, k}(x)\right)=\int f(x) \emptyset_{j, k}(x) d x \\
& d_{j, k}==\left(f(x), \varphi_{j, k}(x)\right)=\int f(x) \varphi_{j, k}(x) d x
\end{aligned}
$$

This algorithm can be implemented in the camera module using a specially designed camera frame based on already defined research that includes the application of CAD, neural networks and IOT [24-26].

\subsection{Discrete 2D Wavelet Transformation of an Image}

In order to facilitate the real-time aspect of this research, a handheld Track Recording Vehicle was developed to which a camera module (see figure 1) was mounted in such a way that it captured the entire surface width of the track. The camera module was connected using the camera pins of the Raspberry Pi 3 B+ (see figure 2).

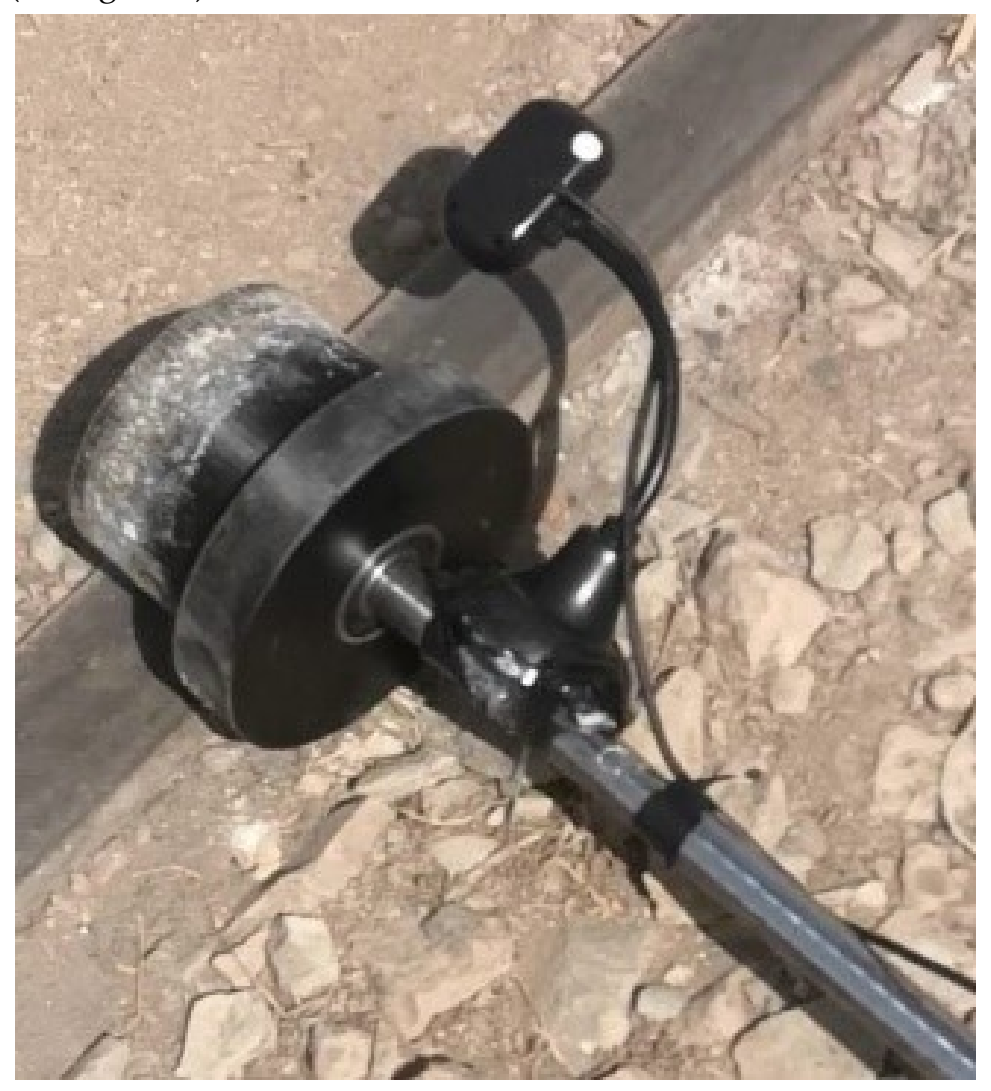

Figure 1. Camera Mounted on Track Recording Vehicle 


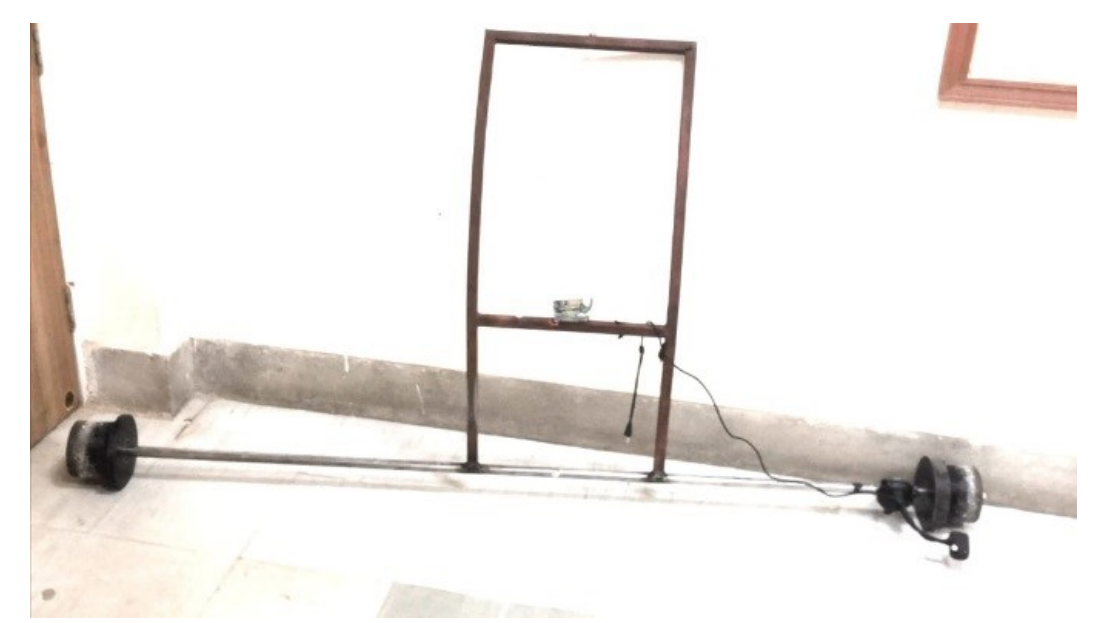

Figure 2. Track Recording Vehicle

\section{Results}

The purpose of this real-time monitoring of the rail track using a handheld track recording vehicle is to avoid railway accidents such as derailment. Using an intact railway track, no damage was detected in the Canny edge detection processed image (see figure 3 (a) and (b)).

(a)

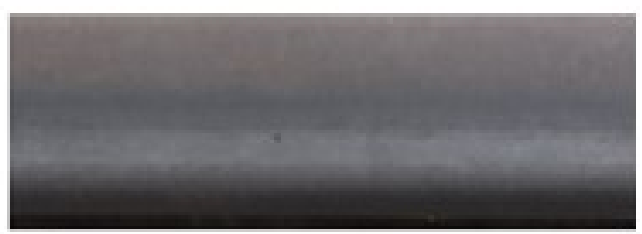

(b)

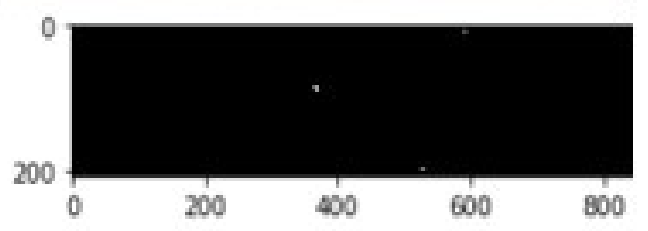

Figure 3. (a) Healthy Track (b) Image Processed from Canny filter

However, when damage started to appear on the track, a visible difference was observed on the Canny edge detection processed image as can be seen in the Fig. 4(a) and (b).

(a)

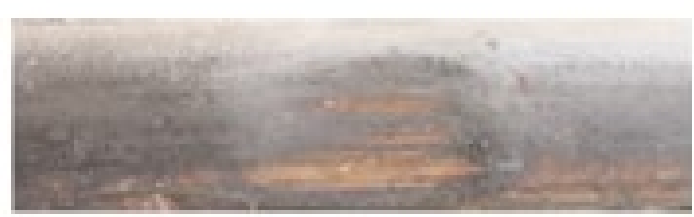

(b)

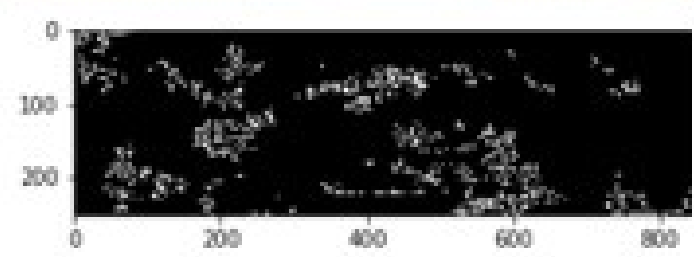

Figure 4. (a) Faulty Track (b) Image Processed from Canny filter

This entire processing was performed using a video feed obtained using the camera module mounted on the track recording vehicle. Thus, the damage (squat as shown in fig.4(a)) that was 
determined using the Canny edge detection algorithm was processed using discrete wavelet transformation. The wavelet transformation further enhances the images as shown in Fig.5 and 6.

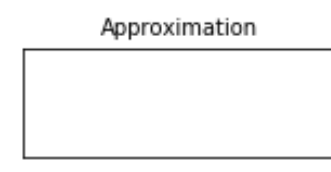

Approximation

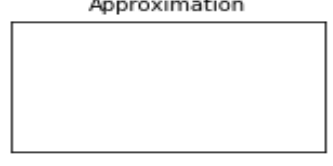

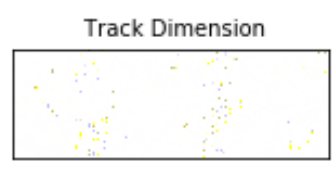
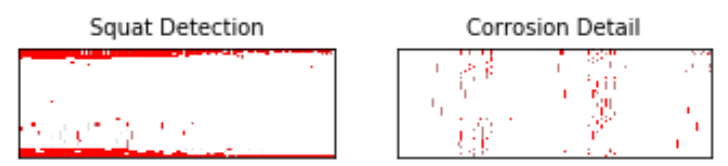

Figure 5. Wavelet Transformation of Healthy Track
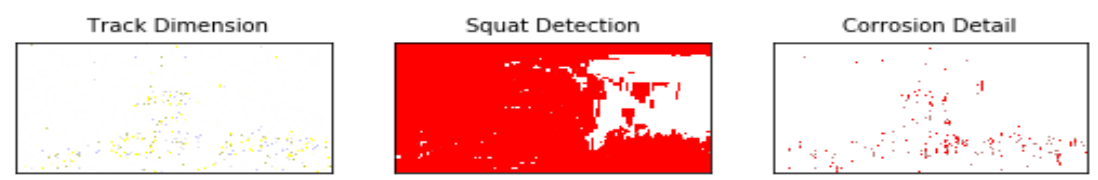

Figure 6. Wavelet Transformation of Faulty Track

A wavelet transformation (images shown in figures 5 and 6) is used to demonstrate the severity of the damage observed on the track and to determine the surface area that is most affected by track damage. As in figure 6, the squat detection demonstrates the area that is most affected by the damage (using the red color, while the white portion represents the unaffected part of the track).

\section{Conclusion}

While wavelet transformation performs better than Canny edge detection in terms of determining the severity of track surface damage, it has processing overheads that become a bottleneck in real-time. The results achieved by the use of the Canny edge detector were found to be encouraging in the determination of the track damage having almost zero processing delay. Around 10,000 various track images were tested and the implementation of this algorithm for the damage detection was validated. This algorithm was tested using a live camera feed that was processed using the attached workstation. The damaged tracks after being analyzed by the Canny edge detection algorithm were captured in the form of images which were later processed using the 2D wavelet transformation. The implementation of this technique has resulted in identifying the damage severity of the track.

Further work is being carried out to develop a real-time neural network algorithm for identifying multiple railway track faults.

\section{Acknowledgements}

This research was funded by the National Center of Robotics and Automation - Condition Monitoring Systems Lab of MUET, Pakistan under HEC grant.

\section{References}

[1] C. Chellaswamy, L. Balaji, A. Vanathi, and L. Saravanan, "IoT based rail track health monitoring and information system", in 2017 International conference on Microelectronic Devices, Circuits and Systems (ICMDCS), 2017, pp. 1-6.

[2] E. J. OBrien, P. Quirke, C. Bowe, and D. Cantero, “Determination of railway track longitudinal profile using measured inertial response of an in-service railway vehicle", Struct. Heal. Monit., vol. 17, no. 6, pp. 14251440, 2018. 
[3] S. Zheng, X. Chai, X. An, and L. Li, "Railway track gauge inspection method based on computer vision", in 2012 IEEE International Conference on Mechatronics and Automation, 2012, pp. 1292-1296.

[4] Hasnain, K., 2020. “A Tough Year For Pakistan Railways: 2019 Marred By Over 100 Accidents”, Available: https://www.dawn.com/news/1525510 [Accessed 20 March 2020].

[5] X. Giben, V. M. Patel, and R. Chellappa, "Material classification and semantic segmentation of railway track images with deep convolutional neural networks", in 2015 IEEE International Conference on Image Processing (ICIP), 2015, pp. 621-625.

[6] A. Malekjafarian, E. OBrien, P. Quirke, and C. Bowe, "Railway Track Monitoring Using Train Measurements: An Experimental Case Study”, Appl. Sci., vol. 9, no. 22, p. 4859, 2019.

[7] A. Malekjafarian, P. J. McGetrick, and E. J. OBrien, “A review of indirect bridge monitoring using passing vehicles", Shock Vib., vol. 2015, 2015.

[8] E. Resendiz, J. M. Hart, and N. Ahuja, “Automated visual inspection of railroad tracks", IEEE Trans. Intell. Transp. Syst., vol. 14, no. 2, pp. 751-760, 2013.

[9] O. Heirich, A. Lehner, P. Robertson, and T. Strang, "Measurement and analysis of train motion and railway track characteristics with inertial sensors", in 2011 14th international IEEE conference on intelligent transportation systems (ITSC), 2011, pp. 1995-2000.

[10] E. G. Berggren, A. Nissen, and B. S. Paulsson, “Track deflection and stiffness measurements from a track recording car", Proc. Inst. Mech. Eng. Part F J. Rail Rapid Transit, vol. 228, no. 6, pp. 570-580, 2014.

[11] R.-K. Liu, P. Xu, Z.-Z. Sun, C. Zou, and Q.-X. Sun, “Establishment of track quality index standard recommendations for Beijing metro", Discret. Dyn. Nat. Soc., vol. 2015, 2015.

[12] M. Molodova, M. Oregui, A. Núñez, Z. Li, and R. Dollevoet, “Health condition monitoring of insulated joints based on axle box acceleration measurements", Eng. Struct., vol. 123, pp. 225-235, 2016.

[13] X. Huang, W. Liu and R. Qin, "A window size selection network for stereo dense image matching", International Journal of Remote Sensing, vol. 41, no. 12, DOI: 10.1080/2150704x.2020.1723168, pp. 4838-4848, 2020.

[14] K. Schröder, M. Rothhardt, W. Ecke, U. Richter, A. Sonntag and H. Bartelt, "Fibre optic sensing system for monitoring of current collectors and overhead contact lines of railways", Journal of Sensors and Sensor Systems, vol. 6, no. 1, DOI: 10.5194/jsss-6-77-2017, pp. 77-85, 2017.

[15] D. Cantero and B. Basu, "Railway infrastructure damage detection using wavelet transformed acceleration response of traversing vehicle", Struct. Control Heal. Monit., vol. 22, no. 1, pp. 62-70, 2015.

[16] X. Zhang, N. Feng, Y. Wang, and Y. Shen, "Acoustic emission detection of rail defect based on wavelet transform and Shannon entropy", J. Sound Vib., vol. 339, pp. 419-432, 2015.

[17] F. Peng, W.-R. Chen, B.-B. Mao, and X.-F. Wang, “Rail-framework abstraction based on Canny edge detection and poly-connection", J. China Railw. Soc., vol. 34, no. 2, pp. 52-57, 2012.

[18] M. Karakose, O. Yaman, M. Baygin, K. Murat, and E. Akin, “A new computer vision based method for rail track detection and fault diagnosis in railways", Int. J. Mech. Eng. Robot. Res., vol. 6, no. 1, pp. 17-22, 2017.

[19] Q. Li, J. Shi, and C. Li, "Fast line detection method for Railroad Switch Machine Monitoring System”, in 2009 International Conference on Image Analysis and Signal Processing, 2009, pp. 61-64.

[20] P. Weston, C. Roberts, G. Yeo, and E. Stewart, "Perspectives on railway track geometry condition monitoring from in-service railway vehicles", Veh. Syst. Dyn., vol. 53, no. 7, pp. 1063-1091, 2015.

[21] L. Gasparetto, S. Alfi, and S. Bruni, “Data-driven condition-based monitoring of high-speed railway bogies”, Int. J. Rail Transp., vol. 1, no. 1-2, pp. 42-56, 2013. 
[22] A. Poulhès, "Dynamic assignment model of trains and users on a congested urban-rail line", Journal of Rail Transport Planning \& Management, DOI: 10.1016/j.jrtpm.2020.100178, p. 100178, 2020.

[23] P. Chatterjee, E. OBrien, Y. Li, and A. González, “Wavelet domain analysis for identification of vehicle axles from bridge measurements", Comput. Struct., vol. 84, no. 28, pp. 1792-1801, 2006.

[24] T. Chetverzhuk, O. Zabolotnyi, V. Sychuk, R. Polinkevych, and A. Tkachuk, “A Method of Body Parts Force Displacements Calculation of Metal-Cutting Machine Tools Using CAD and CAE Technologies", Annals of Emerging Technologies in Computing (AETiC), vol. 3, no. 4, pp. 37-47, DOI: 10.33166/AETiC.2019.04.004, 2019, Available: http://aetic.theiaer.org/archive/v3/v3n4/p4.html.

[25] S. H. Mahmud, L. Assan, and R. Islam, "Potentials of Internet of Things (IoT) in Malaysian Construction Industry", Annals of Emerging Technologies in Computing (AETiC), vol. 2, no. 4, pp. 44-52, DOI: 10.33166/AETiC.2018.04.004, 2018, Available: http://aetic.theiaer.org/archive/v2/v2n4/p4.html.

[26] R. Rudd-Orthner and L. Mihaylova, “Numerical discrimination of the generalisation model from learnt weights in neural networks", Annals of Emerging Technologies in Computing (AETiC), vol. 3, no. 4, pp. 114, DOI: 10.33166/AETiC.2019.04.001, 2019, Available: http://aetic.theiaer.org/archive/v3/v3n4/p1.html.

(C) 2020 by the author(s). Published by Annals of Emerging Technologies in Computing (AETiC), under the terms and conditions of the Creative Commons Attribution (CC BY) license which can be accessed at http://creativecommons.org/licenses/by/4.0. 